ARTICLE

\title{
Haem-activated promiscuous targeting of artemisinin in Plasmodium falciparum
}

Jigang Wang ${ }^{1,2,3,4, \star}$, Chong-Jing Zhang ${ }^{5, \star}$, Wan Ni Chia ${ }^{6}$, Cheryl C.Y. Loh ${ }^{6}$, Zhengjun Li ${ }^{7}$, Yew Mun Lee ${ }^{1}$, Yingke He ${ }^{8}$, Li-Xia Yuan ${ }^{9}$, Teck Kwang Lim${ }^{7}$, Min Liu ${ }^{3}$, Chin Xia Liew ${ }^{7}$, Yan Quan Lee ${ }^{6}$, Jianbin Zhang ${ }^{4}$, Nianci Lu ${ }^{10}$, Chwee Teck Lim¹1,12, Zi-Chun Hua ${ }^{2}$, Bin Liu ${ }^{5}$, Han-Ming Shen ${ }^{4}$, Kevin S.W. Tan ${ }^{6}$ \& Qingsong Lin ${ }^{1,7}$

The mechanism of action of artemisinin and its derivatives, the most potent of the anti-malarial drugs, is not completely understood. Here we present an unbiased chemical proteomics analysis to directly explore this mechanism in Plasmodium falciparum. We use an alkyne-tagged artemisinin analogue coupled with biotin to identify 124 artemisinin covalent binding protein targets, many of which are involved in the essential biological processes of the parasite. Such a broad targeting spectrum disrupts the biochemical landscape of the parasite and causes its death. Furthermore, using alkyne-tagged artemisinin coupled with a fluorescent dye to monitor protein binding, we show that haem, rather than free ferrous iron, is predominantly responsible for artemisinin activation. The haem derives primarily from the parasite's haem biosynthesis pathway at the early ring stage and from haemoglobin digestion at the latter stages. Our results support a unifying model to explain the action and specificity of artemisinin in parasite killing.

\footnotetext{
${ }^{1}$ Department of Biological Sciences, National University of Singapore, Singapore 117543, Singapore. ${ }^{2}$ The State Key Laboratory of Pharmaceutical Biotechnology, College of Life Sciences, Nanjing University, Nanjing 210023, China. ${ }^{3}$ Interdisciplinary Research Group in Infectious Diseases, Singapore-MIT Alliance for Research \& Technology (SMART), Singapore 138602, Singapore. ${ }^{4}$ Department of Physiology, Yong Loo Lin School of Medicine, National University of Singapore, Singapore 117597, Singapore. ${ }^{5}$ Department of Chemical and Biomolecular Engineering, National University of Singapore, Singapore 117585, Singapore. ${ }^{6}$ Department of Microbiology and Immunology, Yong Loo Lin School of Medicine, National University of Singapore, Singapore 117545, Singapore. ${ }^{7}$ NUS Environmental Research Institute, Singapore 117411, Singapore. ${ }^{8}$ Department of Anaesthesiology, Singapore General Hospital, Singapore 169608, Singapore. ${ }^{9}$ School of Traditional Chinese Medicine, Southern Medical University, Guangzhou 510515, China. ${ }^{10}$ College of Life Science and Technology, Beijing University of Chemical Technology, Beijing 100029, China. ${ }^{11}$ Department of Biomedical Engineering, National University of Singapore, Singapore 117583, Singapore. ${ }^{12}$ Department of Mechanical Engineering, National University of Singapore, Singapore 117575, Singapore. ${ }^{*}$ These authors contributed equally to this work. Correspondence and requests for materials should be addressed to J.W. (email: jigang@smart.mit.edu) or to K.S.W.T. (email: kevin_tan@nuhs.edu.sg) or to Q.L. (email: dbslinqs@nus.edu.sg).
} 
P lasmodium falciparum, the most pathogenic human malaria parasite, infects millions of human beings and poses a serious public health threat. Currently, the most potent anti-malarial drugs are artemisinin and its derivatives ${ }^{1,2}$. Artemisinin is a sesquiterpene lactone with an endoperoxide bridge $^{3}$. The hallmark of artemisinin activation is the generation of highly reactive carbon-centred radicals via endoperoxide cleavage $^{4-6}$. Even after several decades of intensive studies on the mechanism of action of artemisinin ${ }^{7,8}$, two critical yet unresolved long-standing questions remain. The first concerns the origin of iron sources required for artemisinin activation. Both free ferrous iron ${ }^{9,10}$ and haem ${ }^{11}$ have been proposed to be the predominant iron sources for its activation, but the results of relevant research are controversial. The second question concerns the exact targets of activated artemisinin. Although several proteins have been reported to be the drug targets $8,9,12$, none of them could satisfactorily account for the quick and highly potent killing effect of artemisinin. It is possible that activated artemisinin might have many other direct targets due to the promiscuous nature of the radicals ${ }^{3,6,8}$. To address these questions, it is critical to develop an approach to directly study the artemisinin-protein interaction.

Here we have developed an unbiased chemical proteomics approach and systematically identified 124 artemisinin covalent binding targets. Many of the identified targets are involved in essential biological processes. We have validated several artemisinin-protein interactions through in vitro binding assays and orthogonal confirmatory experiments. Importantly, targeted enzyme activity was impaired on artemisinin binding in the tested proteins. Thus, our data support the hypothesis that activated artemisinin may kill the malaria parasite through a promiscuous targeting mechanism. In addition, we use in vitro binding analyses to show that haem, rather than free ferrous iron, is predominantly responsible for artemisinin activation. These findings are also supported by an ex vivo cancer cell model. The source of haem required for artemisinin is derived from the parasite's haem biosynthesis pathway at the early ring stage and from haemoglobin digestion at latter stages. Thus, the 'blood-eating' nature of the parasite with the release of extremely high levels of haem confers the high efficacy of artemisinin against the parasites, with minimum side effects towards healthy red blood cells (RBCs). Our results support a unifying model to explain the action and specificity of artemisinin in parasite killing and could facilitate the development of better strategies to treat malaria in times of emerging artemisinin resistance.

\section{Results}

Synthesis of an artemisinin-based activity probe (AP1). To profile and identify the targets of artemisinin, AP1, a chemically engineered artemisinin with a clickable alkyne tag attached ${ }^{13-22}$, was designed and synthesized (Fig. 1a, probe synthesis scheme is shown in Supplementary Fig. 1). The alkyne tag can be further appended with a fluorescent dye or a biotin moiety through click chemistry, allowing the AP1 covalent binding targets to be visualized on SDS-polyacrylamide gel electrophoresis (PAGE) or affinity purified for mass spectrometric identification (Fig. 1b).

Fluorescence labelling of artemisinin targets in parasites. First, we tested the anti-malarial activity of AP1 on two different parasite strains, the laboratory line $P$. falciparum $3 \mathrm{D} 7$ and the field isolate ARS270. AP1 was as potent as the unmodified artemisinin, suggesting that the addition of the clickable alkyne tag does not interfere with drug activity (Fig. 1c and Supplementary Fig. 2). Crude protein extracts were prepared from AP1-treated live parasites, clicked with a fluorescent dye and resolved by
SDS-PAGE. Only AP1 covalent binding targets can be labelled and visualized with fluorescence scanning. Many parasite proteins were fluorescently labelled in an AP1 dose-dependent manner, whereas AP1 failed to react with proteins from healthy RBCs under the same conditions (Fig. 1d). This indicates that AP1 can only be activated inside the parasite but not in the uninfected RBC. We further examined the AP1 labelling of the infected RBCs. The results showed that some of the host cell proteins were also labelled when the RBCs were infected with parasites, although the labelling level was low (Supplementary Fig. 3). This might have been due to the release of parasite-activated drug into the cytosol of infected RBCs. However, this will not cause significant side effects, as the majority of the activated drug is confined within the infected RBCs, as evidenced by the observation that the drug failed to label the uninfected RBCs. Our results ascertained that artemisinin has high specificity towards the malaria parasite and infected RBCs, with minimum side effects towards healthy RBCs. Moreover, AP1 labelling was markedly decreased with preincubation of excess artesunate (a water-soluble artemisinin analogue) (Fig. 1e), suggesting that AP1 binds to the same protein targets as artemisinin. In addition, co-incubation with free-radical scavengers (Tiron, Trolox and TEMPO) also reduced the binding activity of AP1 (Fig. 1f). Collectively, these results indicate that the engineered AP1 is pharmacologically similar to artemisinin. The fluorescence intensity of AP1-labelled proteins on SDS-PAGE serves as a convenient read-out for AP1 binding activity.

Identification of artemisinin targets. Next, we went on to identify the covalent binding targets of AP1. To do so, live parasites (unsynchronized) were incubated for $4 \mathrm{~h}$ with $500 \mathrm{nM}$ AP1, a clinically achievable artemisinin $\operatorname{dose}^{2,23}$, and subjected to crude protein extract preparation, followed by biotin labelling of the alkyne tag. The AP1 targets were affinity purified by streptavidin beads and identified with tandem mass spectrometry. A total of 124 parasite proteins were identified as direct targets of artemisinin in three independent experiments and documented in Supplementary Table 1, while the dimethyl sulfoxide (DMSO)treated control pull-down did not identify any parasite proteins. The statistical analysis and detailed information on the targets are shown in Supplementary Fig. 4 and Supplementary Data 1. Information about the relative abundance of artemisinin targets is shown in Supplementary Fig. 5 and Supplementary Data 1 . Among the proteins in our target list, 33 proteins have previously been developed or proposed as anti-malarial drug targets (Supplementary Table 2). These include SERCA/PfATP6, a previously known artemisinin target ${ }^{9}$.

We further used pull-downs followed by immunoblotting to validate several selected artemisinin targets. Our results confirmed that AP1 could successfully pull-down plasmepsinl (PM I), plasmepsin II (PM II), merozoite surface protein 1 (MSP1) and actin (Supplementary Fig. 6). Gene ontology (GO) analysis revealed that the AP1 targets were involved in many essential biological processes of the parasite, including the carboxylic acid metabolic process, cellular biogenic amine metabolic process, nucleoside metabolic process and ribonucleoside biosynthetic process (Fig. 2). In addition, cellular localization analysis identified several enriched compartments, such as cytosol, vacuolar membrane and notably food vacuoles (Supplementary Fig. 7). Of interest, erythrocyte haemoglobin digestion occurs in the food vacuole ${ }^{24}$ and provides the source of amino acids required to maintain intracellular osmolarity during rapid parasite growth ${ }^{25}$.

Several enzymes involved in the key metabolic pathways of the parasite, as revealed in our GO analysis, including ornithine 


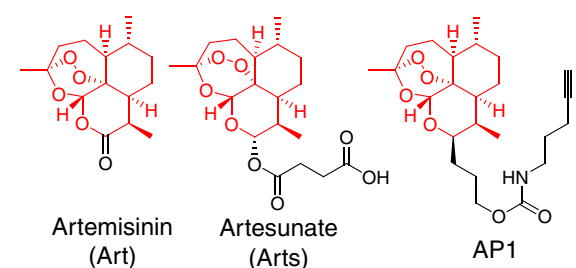

C

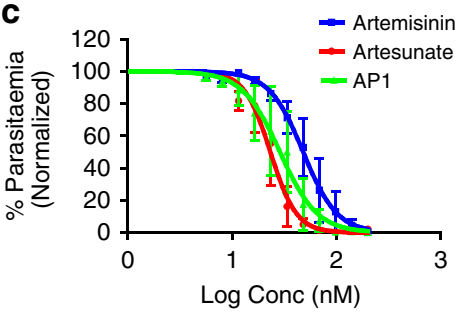

b
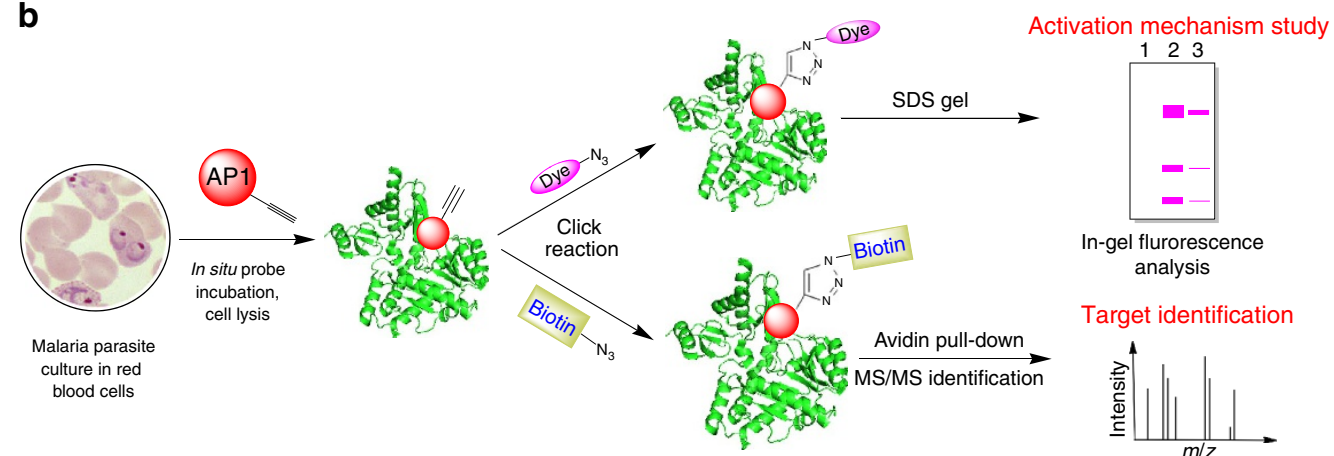

Target identification

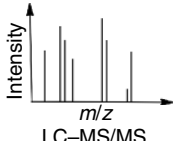

d

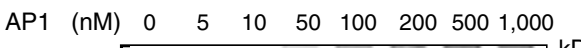

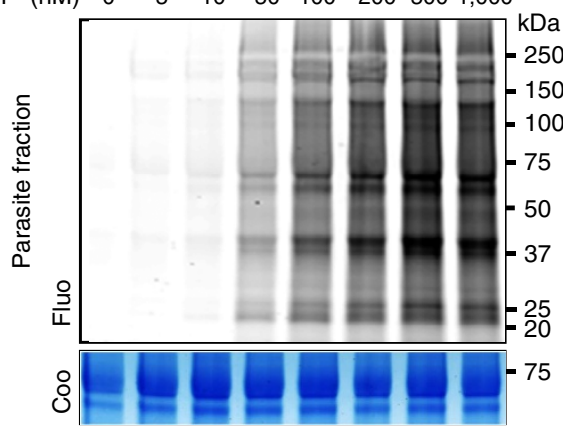

e.

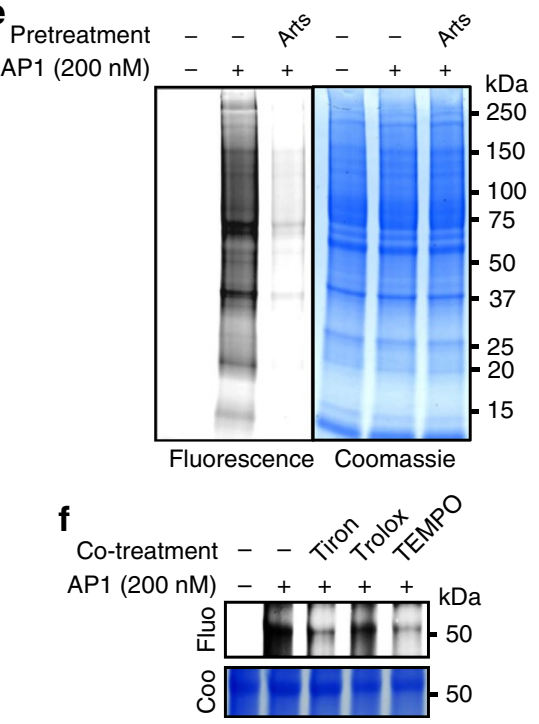

Figure 1 | The chemical proteomics approach to study artemisinin's mechanism of action. (a) Chemical structures of artemisinin (Art), artesunate (Arts) and the alkyne-tagged-clickable probe (AP1). (b) General workflow of the chemical proteomics approach. Fluorescence labelling was used to study the activation mechanism of artemisinin, while biotin pull-downs coupled with LC-MS/MS were used to identify protein targets of artemisinin. (c) The killing effect of AP1 is comparable to that of artemisinin and artesunate on P. falciparum 3D7. (d) In situ parasite labelling with AP1. The labelling was dose dependent and specific to parasite proteins. Healthy RBC cytosolic proteins were not labelled. (e) The AP1 in situ parasite labelling was artemisinin specific as the excess Arts can largely compete with AP1-target labelling. (f) Free-radical scavenger (Tiron, $1 \mathrm{mM}$; Trolox, $400 \mu \mathrm{M}$; TEMPO, $1 \mathrm{mM}$ ) co-treatment reduces the level of parasite protein alkylation by AP1. Fluo, fluorescence scanning; Coo, Coomassie staining. Error bars represent s.d. in three independent replicates in c. Full-gel images for panels $\mathbf{d}$ and $\mathbf{f}$ are shown in Supplementary Fig. 13.

aminotransferase (OAT), pyruvate kinase (PyrK), L-lactate dehydrogenase (LDH), spermidine synthase (SpdSyn) and $S$-adenosylmethionine synthetase (SAMS), were selected to validate their interaction with AP1 in vitro (Table 1). These enzymes were expressed in Escherichia coli, purified and incubated with AP1 in the absence or presence of haem (haemin reduced by L-ascorbic acid $(\mathrm{Vc}))^{26}$. Direct interactions between AP1 and these five enzymes were detected, which were completely thwarted in the presence of excessive amounts of artesunate (Fig. 3a). With OAT as an example, we showed dose- and time-dependent AP1-OAT binding (Fig. 3b,c). The AP1 binding was also protein conformation dependent, as the heat-denatured OAT could not be labelled by AP1 (Fig. 3a). Notably, OAT has been proposed to be a promising anti-malarial target whose inhibitor has a killing effect at the nano-molar range $^{27}$. In addition, PyrK and LDH are involved in the parasite's glycolytic pathway and are responsible for ATP production. Functional assays showed that artesunate inhibits their enzymatic activities in a dose-dependent manner (Fig. 3e,f). Interestingly, translationally controlled tumour protein (TCTP), a previously identified artemisinin target $^{12}$, was not in our target list. This was likely because TCTP contains many lysine and arginine residues, which resulted in extensive trypsin digestion, making it difficult to identify from the artemisinin target mixture (Supplementary 


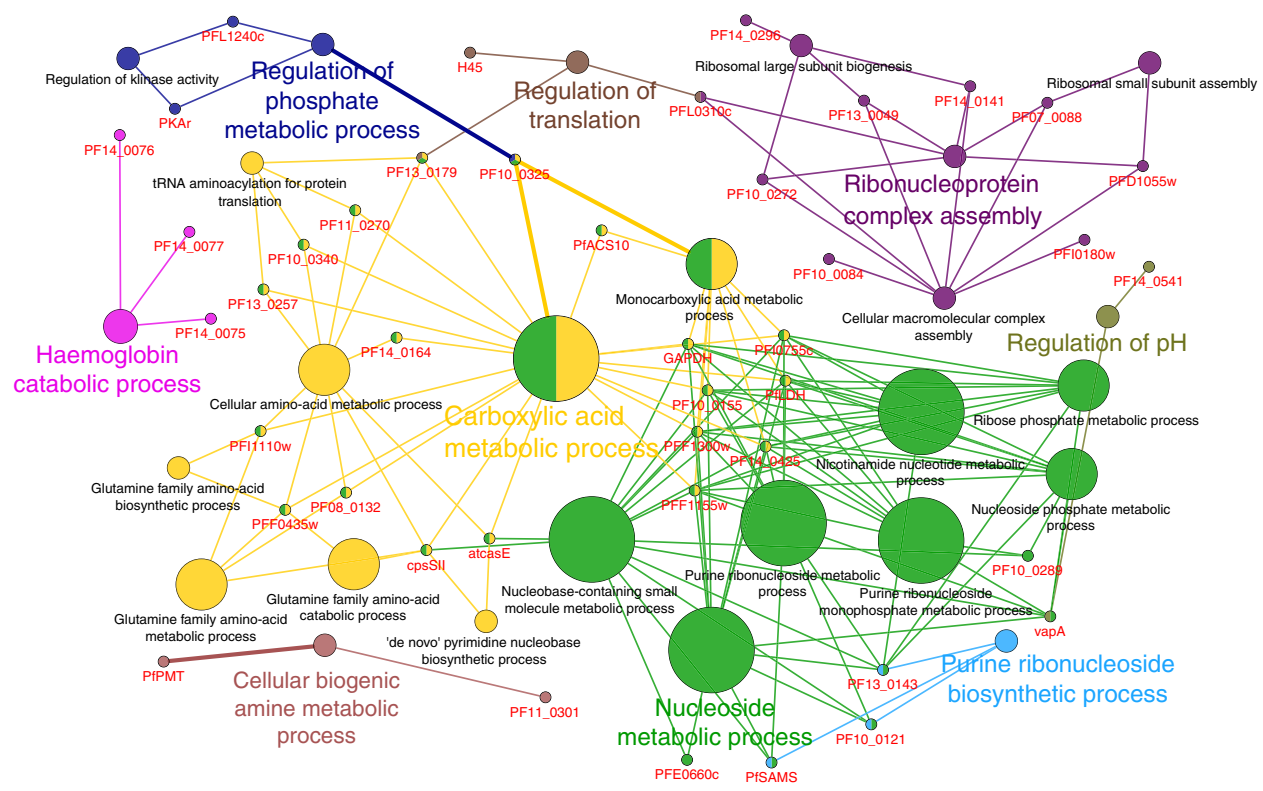

Figure 2 | Artemisinin targets are involved in multiple biological processes essential for parasite survival. GO analysis conducted using Cytoscape with Cluego plugin revealed that the AP1 targets are involved in many essential biological processes of the parasite, including the metabolism of carboxylic acids, cellular biogenic amines and nucleosides, as well as ribonucleoside biosynthesis. This highlights the multi-targeting ability of artemisinin, which exerts numerous effects on the physiological state of the malaria parasite.

\section{Table 1 | Validated artemisinin targets and their functions.}

\section{Target Molecular functions and biological processes involved}

OAT Ornithine metabolism, arginine and proline metabolism

PyrK Glycolysis, pyruvate kinase activity

LDH L-lactate dehydrogenase activity, cysteine and methionine metabolism

SpdSyn Spermidine biosynthesis

SAMS S-adenosylmethionine biosynthesis, methionine adenosyltransferase activity

TCTP* Calcium binding and microtubule stabilization ${ }^{\dagger}$

${ }^{\star}$ TCTP is a known artemisinin target ${ }^{12}$. It was not within the list of our identified artemisinin targets, but it was validated using an in vitro pure protein-binding assay.

The annotated function refers to human TCTP. The function of PfTCTP has not been well characterized.

Table 3). Nevertheless, the AP1-TCTP interaction was also detected by our in vitro assay (Fig. 3a). Furthermore, artemisinin may bind to residues, such as cysteine and lysine, as pretreatment of TCTP with iodoacetamide (IAA) and $N$-ethylmaleimide reduced AP1 labelling (Fig. 3d).

Overall, we systematically identified the artemisinin targets in $P$. falciparum using our newly developed chemical proteomics approach. With artemisinin binding, the key enzymes, including PyrK and LDH, are covalently modified with the bulky sesquiterpene lactone, which irreversibly disrupts the enzymatic activities. The results indicate that artemisinin may kill the parasite through a promiscuous targeting mechanism.

Analysis of the activation mechanism of artemisinin. Our identification of artemisinin targets sheds light on how artemisinin kills the parasite but also provides in vitro models to study how artemisinin is activated, particularly whether haem or free ferrous iron is the prerequisite for drug activation. We found that AP1 itself did not bind to OAT in vitro (Fig. 4a). AP1 binding required the addition of haemin and was further enhanced in the presence of $\mathrm{Vc}, \mathrm{Na}_{2} \mathrm{~S}_{2} \mathrm{O}_{4}$ or glutathione (GSH), reagents that reduce haemin to haem (Fig. 4a). By contrast, the addition of ferrous iron had no detectable effect on AP1-OAT binding (Fig. 4a). The addition of free-iron chelator deferoxamine (DFO) in the presence of haemin or haem only slightly affected AP1-OAT binding (Fig. 4a) ${ }^{28}$.

We therefore reasoned that if haem were indeed the predominant iron source, chelating free ferrous iron should have a minimum effect on AP1-target binding in vivo. As expected, in live parasites (unsynchronized), AP1 binding was essentially unaffected in the absence or presence of DFO (up to $500 \mu \mathrm{M}$, Fig. 4b) or deferiprone (DFP; up to $500 \mu \mathrm{M}$; Supplementary Fig. 8). However, the addition of $N$-acetyl-Leu-Leu-Norleu-al (ALLN), a cysteine protease inhibitor that blocks the digestion of haemoglobin by the parasite to release haem, had a profound inhibitory effect on AP1 binding (Fig. 4b). This was consistent with previous reports that ALLN treatment antagonizes artemisinin and decreases its activity up to 100 -fold ${ }^{11,29}$

Taken together, our results suggest that haem, rather than free ferrous iron, plays a predominant role in artemisinin activation. Previous results have shown that high concentrations of DFP $(500 \mu \mathrm{M})$ can antagonize the potency of artemisinin by increasing the half-maximal inhibitory concentration $\left(\mathrm{IC}_{50}\right)$ value up to fivefold ${ }^{10}$, and this may be explained by the inhibition of iron-related oxidative stress by chelation and by the protection of oxidative damage via iron-independent pathways ${ }^{6,30-34}$.

Activation of artemisinin in an ex vivo cancer model. We also utilized cancer cells as an ex vivo model to further validate the primary roles of haem in artemisinin activation. It has been reported that cancer cells have enhanced haem biosynthesis and are therefore vulnerable to artemisinin ${ }^{35,36}$. As expected, the addition of AP1 covalently modified many proteins in HCT116 colon cancer cells, leading to cell death (Fig. 4c,d). The addition of a haem precursor, $\delta$-aminolevulinic acid (ALA), significantly increased AP1 binding, and its binding was blocked in the presence of the haem synthesis inhibitor succinylacetone (SA) 
a
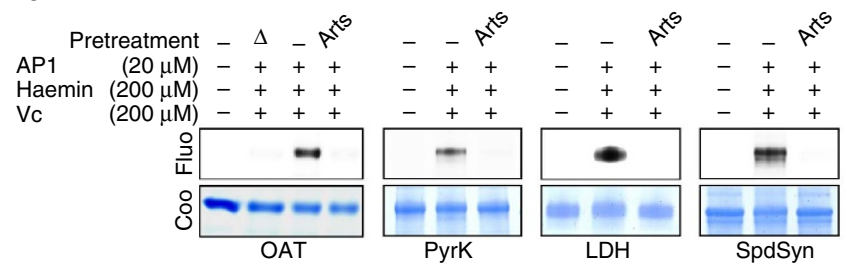

b

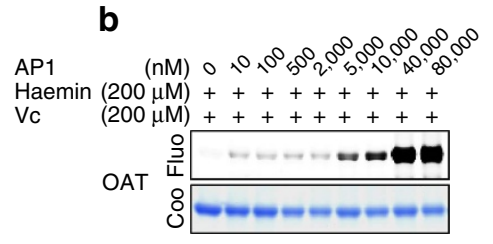

C AP1 $\quad(1 \mu \mathrm{M})+$ oh $0.5 \mathrm{~h}$ th $2 \mathrm{~h} 4 \mathrm{~h} 8 \mathrm{~h}$ Haem

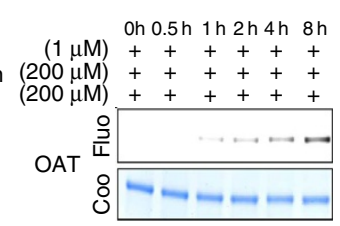

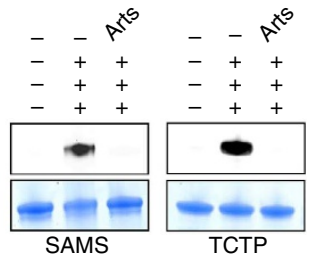

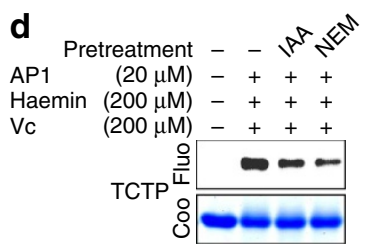

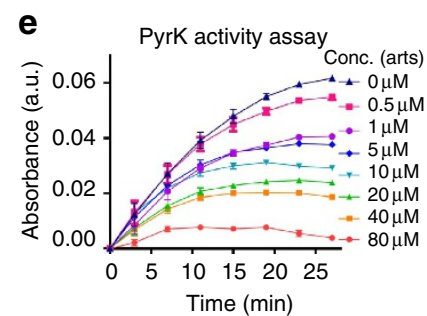

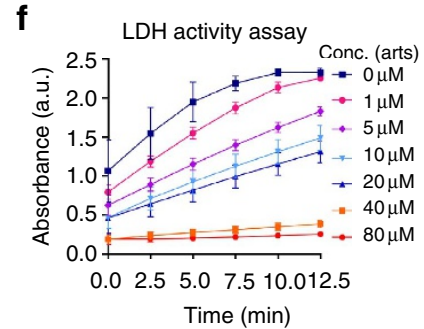

Figure 3 | In vitro binding and functional validation of artemisinin targets. (a) Artemisinin specifically interacts with OAT, PyrK, LDH, SpdSyn, SAMS and TCTP as the unlabelled artesunate $(25 \times)$ can compete with the AP1 binding. Heat denaturation reduces the AP1-labelling level of OAT, suggesting that the interaction of artemisinin with OAT is activity based. (b) Dose-dependent labelling of OAT with AP1 (4h treatment). (c) Time-dependent labelling of OAT with AP1. (d) The interaction of artemisinin with OAT may involve thiol and amine groups as IAA (blocking thiol, $30 \mathrm{mM}$ ) and NEM (blocking amine, $10 \mathrm{mM}$ ) pretreatment $(20 \mathrm{~min})$ can reduce binding. (e,f) Activated artesunate inhibits the activities of PyrK (e) and LDH (f) in vitro. $\Delta$, heat denaturation; IAA, iodoacetamide; NEM, N-ethylmaleimide; Conc., concentration. Error bars represent s.d. in three independent replicates in e and f. Full-gel images for panels a-d are shown in Supplementary Fig. 13.

(Fig. 4c). Consistent with the above results, free ferrous iron had little effect on AP1 binding. The partial inhibitory effect of DFO might have been due to the iron chelation that both inhibits ironrelated oxidative stress and sequesters the iron required for haem synthesis in cancer cells (Fig. 4c) ${ }^{6,30-34}$. Importantly, the extent of AP1 binding in various tested conditions correlated well with its killing effect on cancer cells (Fig. 4e).

Activation of artemisinin at different parasite stages. We next studied artemisinin activation at different asexual intraerythrocytic stages of the parasite. In agreement with the earlier finding that artemisinin is effective against the parasite at different stages ${ }^{37}$, we found that AP1 targeted parasite proteins at all stages, but the effects were smaller against the early ring stage than the latter stages (trophozoite and schizont stages) (Fig. 5a). This is consistent with a previous report ${ }^{38}$ showing that the killing effect of artemisinin is up to 100 times higher at the latter stages than the early ring stage. Unlike the activation of AP1 at the latter stages, the haem source for AP1 activation at the early ring stage was not from haemoglobin digestion ${ }^{29,38}$, as it has not yet occurred at this stage $e^{39}$. This was further confirmed by the observation that ALLN had no inhibitory effect on AP1 activation and binding at the early ring stage (Fig. 5a).

Is the parasite's haem biosynthesis pathway ${ }^{40-44}$ involved in artemisinin activation at the early ring stage? We tested the effects of the haem precursor ALA and the haem synthesis inhibitor SA on artemisinin activation at the early ring stage. The modulation of haem levels through its biosynthetic pathway correlated well with the extent of AP1 activation and binding (Fig. 5b) at the early ring stage, similar to what we observed in the ex vivo cancer model. In addition, the results of ring-stage parasite viability assays showed that ALA pretreatment increased, while SA pretreatment reduced, the parasite-killing effect (Supplementary Fig. 9), consistent with the aforementioned fluorescent-labelling results. Thus, these results reveal a previously unknown mechanism for artemisinin activation in the parasite at the early ring stage. However, when we modulated haem levels using ALA and SA in unsynchronized parasites, there was no significant alteration in the fluorescence-labelling intensity (Supplementary Fig. 10). Furthermore, the $\mathrm{IC}_{50}$ of artesunate co-treated with ALA or SA determined using a standard 48 -h growth inhibition assay were also similar (Supplementary Table 4). Our results are consistent with a previous 5-aminolevulinic acid synthase and ferrochelatase knockout study in P. falciparum ${ }^{45}$, which showed that the modulation of haem biosynthesis does not affect artemisinin activity when the drug treatment spans the entire parasite life cycle in the blood. This also suggests that compared with the level of haem released from haemoglobin, the level of biosynthesized haem is much lower and only plays a minimal role in artemisinin activation compared with haemoglobin digestion at the latter stages. Therefore, we propose that there are two pathways to activate artemisinin in the malaria parasite (Fig. $5 \mathrm{c}$ ). Activation of artemisinin at the early ring stage relies mainly on the parasite's haem biosynthesis; however, the level of activation is rather low due to the low level of haem production by the parasite at this stage. At the latter stages, both of these two pathways may activate artemisinin, but haem released from the digested haemoglobin plays a major role in drug activation. Higher drug activity corresponds to the extremely high haem level at the latter stages (Fig. 5c), which makes artemisinin exceptionally potent against the parasite. This unique mode of artemisinin activation also accounts for the selective toxicity of the drug towards the parasite, as the activated drug is largely confined within the parasites and infected RBCs.

\section{Discussion}

Our results on the mechanisms of artemisinin activation at different parasite stages and the drug's promiscuous targeting are consistent with recent reports of artemisinin resistance ${ }^{46,47}$ due to the PfKelch13 mutation, which results in a prolonged ring stage with an enhanced stress response $23,48,49$. The level of drug activation is low at the ring stage. Parasites with PfKelch13 mutations are able to overcome protein damage due to the drug modifications by activating the stress response; thus, they are selected as they have a higher capability to survive the drug treatment at the ring stage. On the other hand, the much higher 
a

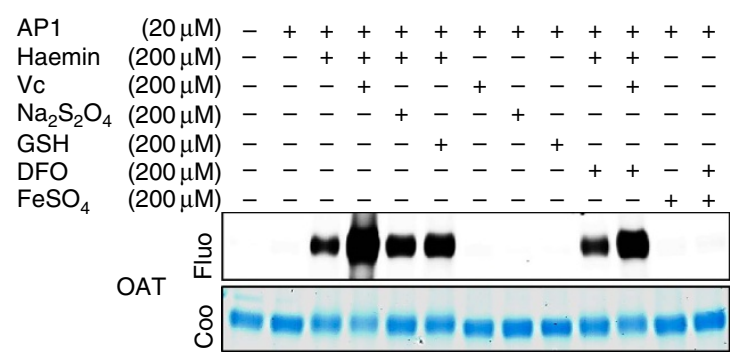

b $_{\text {DFO }}(\mu \mathrm{M})--\quad--100500$

ALLN $(\mu \mathrm{M})-\quad 51015-\quad \mathrm{kDa}$

AP1 $(200 \mathrm{nM})-++++++$

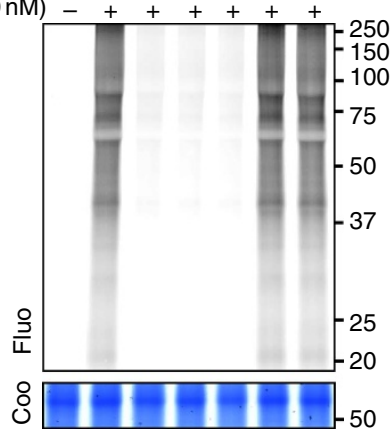

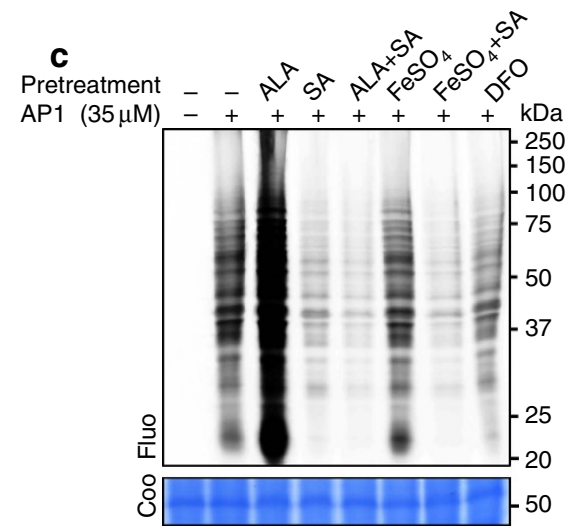

d

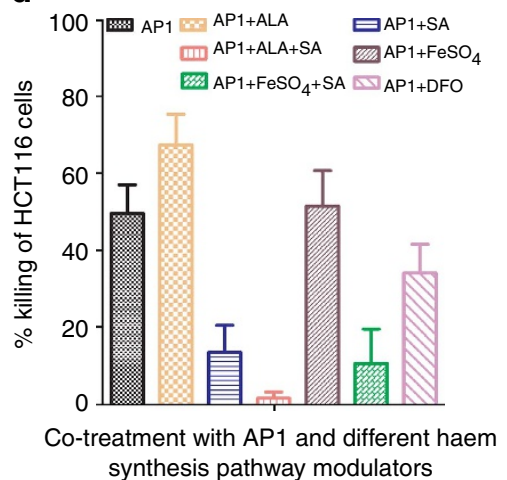

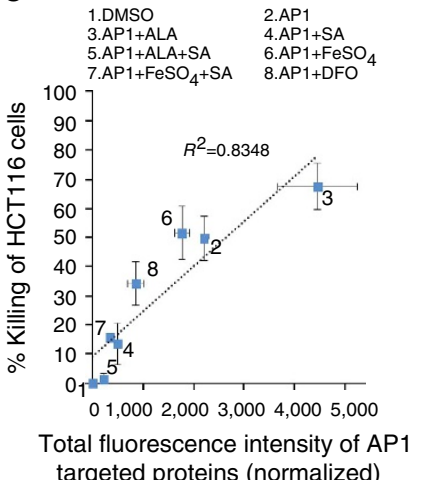

Figure 4 | Artemisinin activation is haem dependent. (a) Artemisinin's interaction with OAT depends mainly on haemin (200 $\mu \mathrm{M})$ or haem (200 $\mu \mathrm{M}$ haemin reduced by L-ascorbic acid $(\mathrm{Vc}, 200 \mu \mathrm{M}), \mathrm{Na}_{2} \mathrm{~S}_{2} \mathrm{O}_{4}(200 \mu \mathrm{M})$ or glutathione $(\mathrm{GSH}, 200 \mu \mathrm{M})$ ) and much less on ferrous iron (FeSO $\left.\mathrm{A}_{4}, 200 \mu \mathrm{M}\right)$. Pretreatment $(30 \mathrm{~min})$ with DFO $(200 \mu \mathrm{M})$ slightly reduced the activation of artemisinin. (b) For the live parasite, pretreatment $(1 \mathrm{~h})$ with the cysteine protease inhibitor ALLN markedly abolished AP1 labelling of the parasite proteins in situ, whereas pretreatment with DFO (30 min) had little effect.

(c) Modulation of the endogenous haem biosynthesis pathway affects the AP1 labelling intensity in HCT116 colon cancer cells. Pretreatment (1 h) with the haem synthesis precursor ALA ( $1 \mathrm{mM})$ enhances the labelling intensity. Conversely, pretreatment with the haem synthesis inhibitor SA (500 $\mu \mathrm{M})$ reduces the labelling intensity. ALA and SA co-treatment did not enhance the labelling intensity as SA inhibition occurs downstream of the haem synthesis pathway. Free iron $\left(\mathrm{FeSO}_{4}, 200 \mu \mathrm{M}\right)$ did not affect probe activation. DFO $(200 \mu \mathrm{M})$ partially reduces the labelling signal intensity, likely due to its role in chelating the free iron, thus impeding the final step of haem synthesis. (d) The percentage of HCT116 cells killed by AP1 treatment or pretreatment with various haem synthesis modulators. (e) Correlation between the AP1 labelling intensity of the total HCT116 target proteins and the percentage of cancer cells killed by AP1 in the presence of various haem synthesis modulators. Error bars represent s.d. in three independent replicates in $\mathbf{d}$ and $\mathbf{e}$. Full-gel images for panels a-c are shown in Supplementary Fig. 13.

level of drug activation at the latter stages triggers extensive protein modifications that act like an exploding bomb, inhibiting multiple key biological processes and eventually resulting in parasite death. Thus, it is less likely that the parasite develops resistance at the latter stages.

Notably, artemisinin resistance due to the PfKelch13 mutation is different from typical drug resistance caused by mutations in drug targets. The multi-targeting nature of artemisinin makes it difficult for $P$. falciparum to acquire resistance due to the need for mutations of multiple targets. Thus, it is not surprising that previous studies could not find the association of PfATP6 and TCTP (known targets) mutations with the emerging drug resistance ${ }^{50}$. Rather, PfKelch13 mutations enhance the parasites' capability to cope with the stress induced by artemisinin multitargeting at the ring stage, at which point drug activation and drug pressure are relatively low; thus, these mutations can be selected.

To cope with artemisinin resistance due to a prolonged ring stage, we can either extend the length of drug treatment, as suggested by Tilley and co-workers ${ }^{51}$, or increase the drug's activation level at the ring stage. As we have shown, pretreatment with ALA enhances artemisinin activation. ALA has been widely used in photodynamic therapy, such as the treatment of skin cancer, for many years ${ }^{52}$. Thus, co-treating patients with both ALA and artemisinin might improve the drug's efficacy towards ring-stage parasites.

Our results indicate that the clickable artemisinin probe is useful for the identification of protein targets of the drug and the visualization of drug activation levels both in vitro and in vivo. However, our experimental conditions are not useful for identifying non-covalent targets. Recently, a reversible artemisinin target, PfPI3K, was reported to be related to drug resistance in the ring stage ${ }^{53}$. The existence of non-covalent artemisinin targets, possibly with many others yet to be identified, further supports the notion that artemisinin acts via a promiscuous targeting mechanism. It will be interesting to develop new artemisinin probes that can be used to capture transient artemisinin-protein interactions. It will also be interesting to determine the roles of each covalent and noncovalent binding target in artemisinin-mediated parasite killing in future studies.

We have demonstrated here that artemisinin activation is mainly haem dependent, which we believe resolves previous arguments regarding the source of iron required for the drug activation. Artemisinin activation at the early ring stage seems to 
a

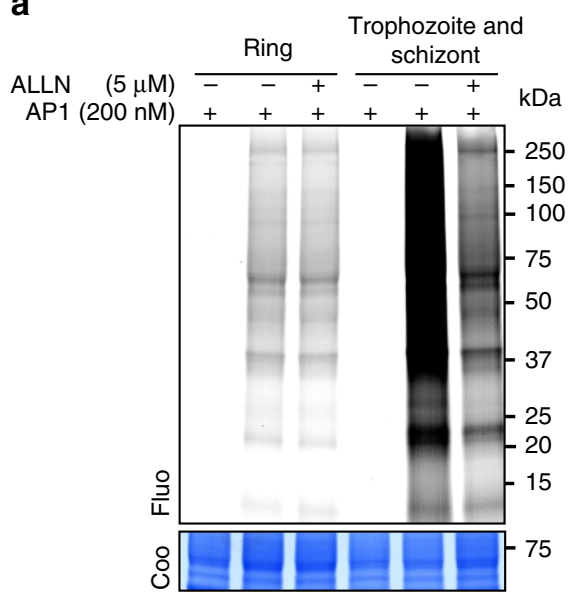

b

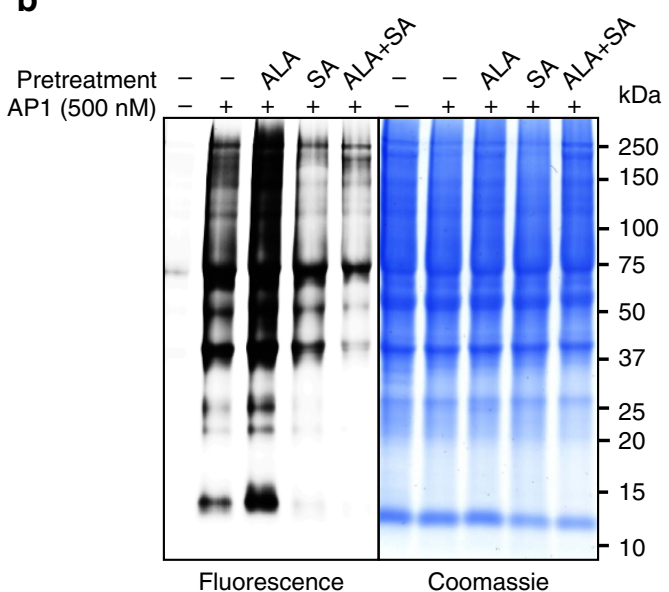

C

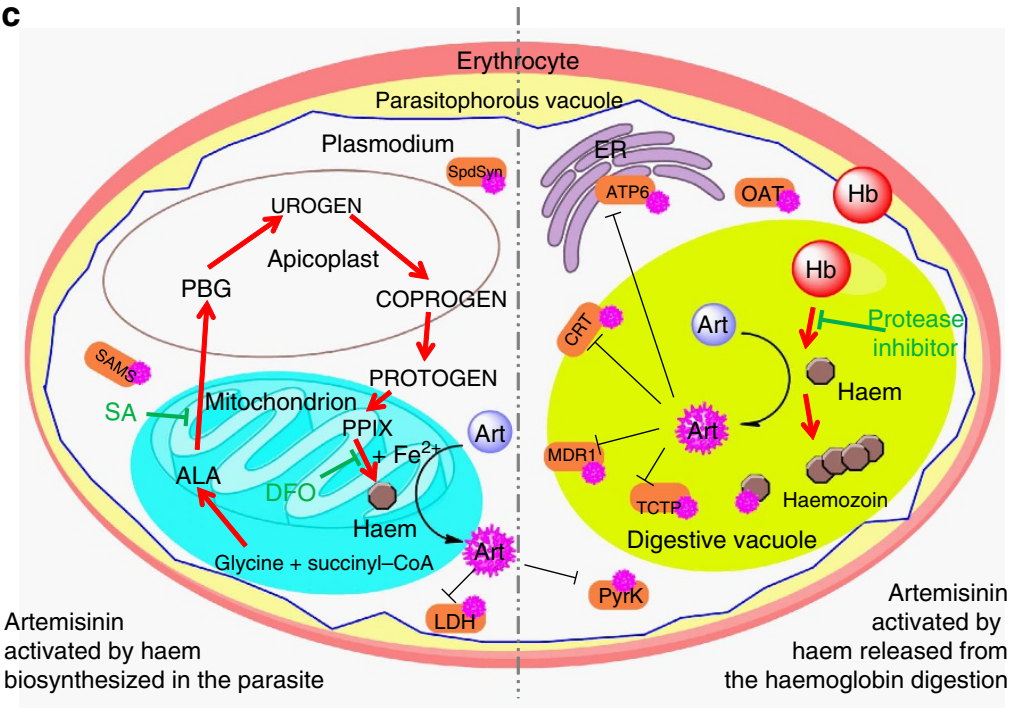

Figure 5 | Artemisinin activation in P. falciparum relies on two distinct haem sources. (a) AP1 fluorescence-labelling intensity is much lower at the early ring stage compared with the latter stages (trophozoite and schizont stages) of the parasite. The cysteine protease inhibitor ALLN reduced the labelling intensity at the latter stages but could not reduce the labelling intensity at the early ring stage. (b) Modulation of the haem biosynthesis pathway of the parasite affected the labelling intensity of AP1 in the early ring stage. Pretreatment (1 h) with the haem synthesis precursor ALA (1 mM) enhanced the labelling signal intensity. Conversely, pretreatment with the haem synthesis inhibitor SA $(500 \mu \mathrm{M})$ partially reduced the labelling intensity. (c) A model for artemisinin's mechanism of action. Artemisinin activation relies on haem generated in the parasite from both biosynthesis and haemoglobin digestion. In the early ring stage, biosynthesized haem was primarily responsible for drug activation; at the latter stages, both pathways co-existed, with haem derived from haemoglobin digestion playing the major role. COPROGEN, coproporphyrinogen III; CRT, chloroquine resistance transporter; ER, endoplasmic reticulum; Hb, haemoglobin; MDR, multidrug resistance protein; PBG, porphobilinogen; PPIX, protoporphyrin IX; PROTOGEN, protoporphyrinogen IX UROGEN, uroporphophyrinogen III. Full-gel images for panels $\mathbf{a}$ and $\mathbf{b}$ are shown in Supplementary Fig. 13.

rely on the parasite's haem biosynthesis, whereas drug activation depends on haemoglobin digestion as the main haem source at the latter parasite stages, which determines the high specificity of the drug towards the parasite. Our findings contribute to a better understanding of the mechanism of action of artemisinin, which may facilitate the development of better strategies to fight malaria and its emerging drug resistance.

\section{Methods}

Synthesis of AP1. Reagents were obtained commercially and were used without further purification, except where indicated. Dichloromethane and 1,2-dichloroethane were freshly distilled from calcium hydride under a nitrogen atmosphere. Tetrahydrofuran was dried by distillation with sodium as a drying agent and benzophenone as an indicator. Silica gel (230-400 mesh) was used in flash column chromatography. ${ }^{1} \mathrm{H}$ and ${ }^{13} \mathrm{C}$ NMR spectra were recorded on a Bruker ARX 300. Chemical shifts ( $\delta$ p.p.m.) were determined with tetramethylsilane as the internal reference. Mass spectra were recorded on a Finnigan LCQ mass spectrometer.

The synthetic scheme is shown in Supplementary Fig. 1. The preparation of intermediate 1 follows previously described methods ${ }^{54}$ with slight modifications. $\mathrm{NaBH}_{4}(1.2 \mathrm{~g}, 32.0 \mathrm{mmol})$ was added to a stirred solution of artemisinin $(6.0 \mathrm{~g}, 21.2 \mathrm{mmol})$ in $\mathrm{CH}_{3} \mathrm{OH}(50 \mathrm{ml})$ at $0{ }^{\circ} \mathrm{C}$. After the resulting mixture was stirred at the same temperature for $3 \mathrm{~h}$, the mixture was neutralized with glacial acetic acid while maintaining the temperature at $0{ }^{\circ} \mathrm{C}$. The mixture was then concentrated by evaporating most of the $\mathrm{CH}_{3} \mathrm{OH}$, and the resulting residue was diluted with cold water and stirred for $15 \mathrm{~min}$ at room temperature. The precipitate was collected, washed with water and dried to give the title compound as a white solid $(5.0 \mathrm{~g}, 85.0 \%$ yield). ${ }^{1} \mathrm{H}$ NMR $\left(300 \mathrm{MHz}, \mathrm{CDCl}_{3}\right) \delta 5.60-5.48(\mathrm{~m}, 1 \mathrm{H}), 5.30-4.73(\mathrm{~m}, 1 \mathrm{H})$, 2.80-2.61 (m, 1H), 2.38-2.37 (m, 1H), 1.86-1.48 (m, 8H), 1.44-1.43 (m, 3H), $1.30-1.26(\mathrm{~m}, 3 \mathrm{H}), 0.97-0.89(\mathrm{~m}, 6 \mathrm{H})$; liquid chromatography-mass spectrometry (LC-MS; APCI) calcd for $\left[\mathrm{M}+\mathrm{H}-\mathrm{H}_{2} \mathrm{O}\right]^{+}: 267.15$, found: 267.10 .

The preparation of intermediate 2 followed previous descriptions ${ }^{55}$ with slight modifications. Benzoyl chloride $(3.2 \mathrm{ml}, 27.6 \mathrm{mmol})$ was added to a stirred solution of dihydroartemisinin $1(5.0 \mathrm{~g}, 17.6 \mathrm{mmol})$ in anhydrous dichloromethane $(60 \mathrm{ml})$ and anhydrous pyridine $(9.0 \mathrm{ml})$ at $0^{\circ} \mathrm{C}$. After stirring at room temperature for 
$16 \mathrm{~h}$, citric acid solution ( $7 \%$ in water, $50 \mathrm{ml}$ ) was added. The organic layer was separated, and the aqueous layer was further extracted with ethyl acetate $(10 \mathrm{ml})$. The combined organic layer was washed with citric acid solution (7\% in water, $50 \mathrm{ml}$ ), followed by saturated $\mathrm{NaHCO}_{3}$ aqueous solution $(50 \mathrm{ml})$ and water $(50 \mathrm{ml})$. The organic layer was then dried and concentrated, and the residue was purified by column chromatography (hexane:ethyl acetate $=10: 1$ ) to yield the title compound as a white solid $\left(6.0 \mathrm{~g}, 88.0 \%\right.$ yield). ${ }^{1} \mathrm{H} \mathrm{NMR}\left(300 \mathrm{MHz}, \mathrm{CDCl}_{3}\right) \delta 8.13(\mathrm{~m}, 2 \mathrm{H})$, 7.57-7.45(m, 3H), $6.02(\mathrm{~m}, 1 \mathrm{H}), 5.53(\mathrm{~s}, 1 \mathrm{H}), 2.76(\mathrm{~m}, 1 \mathrm{H}), 2.40(\mathrm{~m}, 1 \mathrm{H}), 2.08-0.93$ (m, 19H), 0.99-0.98 (m, 3H), 0.93-0.92 (m, 3H); LC-MS (APCI) calcd for $[\mathrm{M}+\mathrm{Na}]^{+}: 411.18$, found: 411.20 .

The preparation of intermediate 3 followed previously described methods ${ }^{56,57}$ with slight modifications. A solution of intermediate $2(4.2 \mathrm{~g}, 11.0 \mathrm{mmol})$ in anhydrous 1,2-dichloroethane $(50 \mathrm{ml})$ was added dropwise via cannula to a stirred mixture of allyltrimethylsilane $(8.8 \mathrm{ml}, 56 \mathrm{mmol})$, anhydrous $\mathrm{ZnCl}_{2}(1.8 \mathrm{~g}$, $13.2 \mathrm{mmol}$ ) and $4 \mathrm{~A}$ molecular sieve powder in anhydrous 1,2 -dichloroethane $(50 \mathrm{ml})$ at $0{ }^{\circ} \mathrm{C}$. After stirring at the same temperature for $3 \mathrm{~h}$, the reaction mixture was diluted with ethyl acetate $(300 \mathrm{ml})$ and washed with citric acid solution $(5 \%$ in water, $100 \mathrm{ml})$, saturated $\mathrm{NaHCO}_{3}$ aqueous solution $(100 \mathrm{ml})$ and brine $(100 \mathrm{ml})$. The organic layer was dried and concentrated. The residue was purified by column chromatography (hexane:ethyl acetate $=10: 1$ ) to give the key intermediate as a white solid $(2.5 \mathrm{~g}, 75.0 \%)$. Next, $\mathrm{BH}_{3} \mathrm{SMe}_{2}$

(borane dimethylsulfide; $2.0 \mathrm{M}$ in diethyl ether, $2.0 \mathrm{ml}, 4.0 \mathrm{mmol}$ ) was added to a stirring solution of this key intermediate $(1.04 \mathrm{~g}, 3.36 \mathrm{mmol})$ in anhydrous tetrahydrofuran $(20 \mathrm{ml})$ at $0{ }^{\circ} \mathrm{C}$. The reaction mixture was allowed to warm up to room temperature and stirred for $24 \mathrm{~h}$. A suspension of $\mathrm{NaBO}_{3} \cdot 4 \mathrm{H}_{2} \mathrm{O}$ $(2.50 \mathrm{~g}, 16.25 \mathrm{mmol})$ in water $(25 \mathrm{ml})$ was then slowly added to the reaction mixture, and the resulting suspension was stirred for $24 \mathrm{~h}$. Water $(25 \mathrm{ml})$ was added, and the mixture was extracted with $\mathrm{CH}_{2} \mathrm{Cl}_{2}(25 \mathrm{ml})$. The organic extracts were dried over $\mathrm{Na}_{2} \mathrm{SO}_{4}$, filtered and concentrated under reduced pressure to give a colourless oil, which was then purified by column chromatography (hexane:ethyl acetate $=4: 1$ to hexane:ethyl acetate $=2: 1)$ to obtain intermediate $3(0.66 \mathrm{~g}, 62.0 \%$ yield) as a white solid. ${ }^{1} \mathrm{H}$ NMR $\left(300 \mathrm{MHz}, \mathrm{CDCl}_{3}\right) \delta 5.53(\mathrm{~s}, 1 \mathrm{H}), 4.24-4.23$ (m, 1H), 3.73-3.67 (m, 2H), 2.65-2.64 (m, 1H), 2.33-2.29 (m, 2H), 2.06-1.19 $(17 \mathrm{~m}, 3 \mathrm{H}), 0.97-0.80(6 \mathrm{~m}, 3 \mathrm{H})$

A solution of intermediate $3(326 \mathrm{mg}, 1.0 \mathrm{mmol})$, hex-5-ynoic acid $(38 \mathrm{mg}$, $0.37 \mathrm{mmol})$, diphenylphosphoryl azide $(275 \mathrm{mg}, 1.0 \mathrm{mmol})$ and $\mathrm{Et}_{3} \mathrm{~N}(101 \mathrm{mg}$, $1.0 \mathrm{mmol})$ in anhydrous $\mathrm{CH}_{3} \mathrm{CN}(50 \mathrm{ml})$ was stirred at $55^{\circ} \mathrm{C}$ overnight under nitrogen. The reaction mixture was allowed to cool to room temperature, and the solvent was removed under reduced pressure. The resulting residue was taken up in ethyl acetate $(100 \mathrm{ml})$ and washed with citric acid $(5 \%$ in water, $50 \mathrm{ml} \times 2)$, water $(50 \mathrm{ml})$, saturated $\mathrm{NaHCO}_{3}$ aqueous solution $(50 \mathrm{ml})$ and brine $(50 \mathrm{ml})$. The organic extracts were dried over $\mathrm{Na}_{2} \mathrm{SO}_{4}$, filtered and concentrated to give a colourless oil, which was then purified by flash chromatography (hexane:ethyl acetate $=7: 3)$ to obtain AP1 $\left(90 \mathrm{mg}, 58.0 \%\right.$ yield) as a white solid. ${ }^{1} \mathrm{H}$ NMR $\left(300 \mathrm{MHz}, \mathrm{CDCl}_{3}\right) \delta 5.29(\mathrm{~s}, 1 \mathrm{H}), 4.79(\mathrm{~m}, 1 \mathrm{H}), 4.18-4.10(\mathrm{~m}, 3 \mathrm{H}), 3.30-3.28$ (m, $2 \mathrm{H}), 2.67-2.64(\mathrm{~m}, 1 \mathrm{H}), 2.43-2.25(\mathrm{~m}, 4 \mathrm{H}), 2.06-1.73(\mathrm{~m}, 5 \mathrm{H}), 170-1.56$ (m, 8H), 1.40-1.25 (m, 5H),0.97-0.95 (m, 3H), 0.87-0.85 (d, 3H) (see Supplementary Fig. 11); high-resolution mass spectrometry (ESI) calculated for $[\mathrm{M}+\mathrm{H}]^{+}:$: 436.2694, found: 436.2700 (see Supplementary Fig. 12).

Reagents and equipment used in biological experiments. In addition to the aforementioned synthesized chemicals, other reagents used in our biological experiments are listed below. Artemisinin, artesunate, streptavidin beads, methanol, acetonitrile (ACN), formic acid (FA), urea, phosphoric acid, DMSO, Tris [(1-benzyl-1H-1,2,3-triazol-4-yl) methyl]amine (TBTA), Tris (2-carboxyethyl) phosphine (TCEP), $\mathrm{CuSO}_{4}, \mathrm{ALLN}$, haemin, L-ascorbic acid (vitamin C), $\mathrm{Na}_{2} \mathrm{SO}_{4}$, DFO, DFP, $\mathrm{FeSO}_{4}$, (IAA, $\mathrm{N}$-ethylmaleimide, ALA, SA, 4,5-dihydroxy-1,3-benzenedisulfonic acid disodium salt (Tiron), 6-hydroxy-2,5,7,8-tetramethylchroman-2carboxylic acid (Trolox), 2,2,6,6-tetramethyl-1-piperidinyloxy (TEMPO), LDH and PyrK assay kits were purchased from Sigma-Aldrich (St Louis, MO, USA). Rhodamine-azide was obtained from Jinglan Co. (Guangzhou, China). Methyl methanethiosulfonate (MMTS) was purchased from Pierce (Rockford, IL, USA). RPMI 1640, Dulbecco's modified eagle medium and BSA were purchased from Invitrogen (Carlsbad, CA, USA). Sequencing grade trypsin was obtained from Promega (Madison, WI, USA). Protease and phosphatase inhibitor cocktails were purchased from Roche (Basel, Switzerland). All the parasite cultures were maintained in malaria culture media (MCM) consisting of RPMI 1640 (Invitrogen, USA) supplemented with 0.5\% Albumax II (Gibco, Auckland, New Zealand), $0.25 \%$ gentamycin (Gibco, USA), 0.005\% hypoxanthine (Sigma-Aldrich), $0.03 \%$ L-glutamate (Sigma-Aldrich) and $1.25 \%$ healthy human erythrocytes (Interstate Blood Bank, USA). Unless otherwise indicated, all other reagents used for the biochemical methods were purchased from Sigma-Aldrich. In-gel fluorescence scanning of SDS-PAGE gels was carried out with a Typhoon 9410 fluorescence scanner (GE Healthcare; Buckinghamshire, UK), and where applicable, the gel lanes were quantified using ImageQuant (GE Healthcare).

Parasite culture and synchronization. P. falciparum laboratory strain 3D7 (MRA-102, MR4/ATCC (American Type Culture Collection), Manassas, VA, USA and Thailand-derived field isolate ARS 270 (a kind gift from François Nosten,
Shoklo Malaria Research Unit (SMRU), Mae Sot, Thailand) were continuously cultured in the above-mentioned MCM culture medium. Culture flasks were gassed with $3 \% \mathrm{CO}_{2}, 4 \% \mathrm{O}_{2}$ and $93 \% \mathrm{~N}_{2}$, and incubated at $37^{\circ} \mathrm{C}$. Synchronization of parasite cultures was performed by incubating the cultures in $5 \%(w / v)$ D-sorbitol (Merck, Germany) at $37^{\circ} \mathrm{C}$ for $10 \mathrm{~min}$, after which the cells were washed twice with culture medium before transferring to new flasks. Synchronization will lyse erythrocytes containing mature stages of parasites (trophozoites and schizonts) through hypotonic solution, leaving the ring stage-infected and -uninfected erythrocytes in the culture. Giemsa-stained thin blood smears were examined before each experiment to check for parasitaemia and parasite stages.

Inhibitory concentration determination. Synchronized ring-stage cultures were diluted with fresh erythrocytes and MCM to $1 \%$ parasitaemia and $1.25 \%$ haematocrit. The cultures were then incubated with artemisinin, artesunate or AP1 at different concentrations for $48 \mathrm{~h}$ in a 96 -well plate. Subsequently, the cells were stained with $1 \mu \mathrm{g} \mathrm{ml}^{-1}$ of Hoechst 33342 (Invitrogen) for $20 \mathrm{~min}$ at $37^{\circ} \mathrm{C}$ in the dark, and parasitaemia was determined with a CyAn flow cytometer (Beckman Coulter). Plotting of the sigmoidal dose-response curve was performed with GraphPad Prism 5 using a four-parameter logistic curve (variable slope). Three experiments were performed to obtain the mean values presented.

Modified ring-stage viability assay. Tightly synchronized ring-stage ( $0-6 \mathrm{~h})$ cultures were diluted with fresh erythrocytes and MCM to $1 \%$ parasitaemia and $1.25 \%$ haematocrit. The cultures were then incubated with PBS, SA $(500 \mu \mathrm{M})$ or ALA ( $1 \mathrm{mM})$ for $1 \mathrm{~h}$ in a six-well plate at $37^{\circ} \mathrm{C}$. Subsequently, the cells were aliquoted into 96-well plates and then incubated with varying concentrations of artesunate $(0-500 \mathrm{nM})$ for another $4 \mathrm{~h}$ at $37^{\circ} \mathrm{C}$. Finally, the drugs were washed off three times with fresh malaria culture medium and then returned to the $37^{\circ} \mathrm{C}$ incubator for another $43 \mathrm{~h}$. Cells were stained with $1 \mu \mathrm{g} \mathrm{ml}^{-1}$ of Hoechst 33342 (Invitrogen) for $20 \mathrm{~min}$ at $37^{\circ} \mathrm{C}$ in the dark, and the parasitaemia was determined with a CyAn flow cytometer (Beckman Coulter). Plotting of the sigmoidal dose-response curve was performed with GraphPad Prism 5 using a fourparameter logistic curve (variable slope). Three experiments were performed to obtain the mean values presented.

In situ fluorescence labelling of $\boldsymbol{P}$. falciparum. P. falciparum parasites (unsynchronized) were diluted to $\sim 5 \%$ parasitemia and cultured with $1.25 \%$ haematocrit in a 12-well plate $(1 \mathrm{ml}$ parasite culture) in the gassed incubation chamber. Increasing concentrations $(5-1,000 \mathrm{nM})$ of AP1 dissolved in DMSO (final concentration of DMSO was $0.2 \%$ ) were used to culture the parasites for $4 \mathrm{~h}$ at $37^{\circ} \mathrm{C}$. An equal volume of DMSO was used as the negative control. After probe incubation, the cultures were transferred to Eppendorf tubes and centrifuged at $600 \mathrm{~g}$ for $5 \mathrm{~min}$ to remove the medium. Subsequently, $0.05 \%$ saponin in PBS was added, and the tubes were inverted several times before incubating on ice for $10 \mathrm{~min}$ to lyse RBCs. The cell lysates were then centrifuged at 10,000 r.p.m. $(9,300 \mathrm{~g})$ for $10 \mathrm{~min}$ to remove $\mathrm{RBC}$ proteins. The resulting pellets were washed with PI buffer $(1 \times$ protease inhibitors in PBS) and centrifuged at 10,000 r.p.m. $(9,300 g)$ to collect the parasite fractions. To ensure removal of the RBC proteins, pellets were further washed twice with the same buffer. Parasite proteins were extracted by suspending the pellets in $0.16 \%$ SDS in PBS with $1 \times$ protease inhibitors (EDTA free, Roche) before a brief sonication ( $31 \%$ amplification, $2 \mathrm{~s}$ on, $2 \mathrm{~s}$ off for $2 \mathrm{~min}$ on ice). Clear lysates were obtained via centrifugation $(9,300 \mathrm{~g})$, and the protein concentrations were determined by DC protein assay (Bio-Rad). Equal amounts $(50 \mu \mathrm{g})$ of different treatment samples were used for fluorescent tagging. For each click reaction, rhodamine-azide $(10 \mu \mathrm{M})$, TBTA ligand $(100 \mu \mathrm{M}, 100 \times$ stock in DMSO), TCEP $(1 \mathrm{mM}, 100 \times$ fresh stock in water $)$ and $\mathrm{CuSO}_{4}(1 \mathrm{mM}, 100 \times$ stock in water) were sequentially added to the lysates. Samples were incubated and shaken at room temperature for $3 \mathrm{~h}$. Next, tagged proteins were precipitated with acetone and air dried. $1 \times$ Laemmli buffer $(35 \mu \mathrm{l})$ was added to dissolve the pellets, and $15 \mu \mathrm{l}$ of each sample was separated by SDS-PAGE on $12.5 \%$ polyacrylamide gels or $4-20 \%$ gradient gels (Bio-Rad). Following one-dimensional gel separation, gels were scanned using a Typhoon 9410 laser scanner (GE Healthcare), and the images were analysed with ImageQuant software. The fluorescence contrast was normalized against the DMSO control to minimize the background.

For competition assays, the parasites were pretreated with excessive artesunate $(25 \times)$ for $30 \mathrm{~min}$ then together with AP1 $(200 \mathrm{nM})$ for $4 \mathrm{~h}$. Probe-labelled proteins were visualized by click conjugation to the rhodamine-azide followed by SDS-PAGE separation and fluorescence scanning.

For free-radical scavenger co-treatment, the parasites were co-treated with various scavengers together with AP1 for $4 \mathrm{~h}$. The concentrations of the free-radical scavengers used in this experiment were: Tiron $(1 \mathrm{mM})$, Trolox $(400 \mu \mathrm{M})$ and TEMPO $(1 \mathrm{mM})$. Similarly to the aforementioned fluorescent labelling, drug- or chemical-treated parasites were isolated from RBC and lysed. The probe-labelled proteins were then subjected to rhodamine-azide conjugation, SDS-PAGE separation and fluorescence scanning.

For DFO, DFP and ALLN pretreatment, the parasites were pretreated with different concentrations of DFO or DFP (100 or $500 \mu \mathrm{M}, 30 \mathrm{~min})$ or ALLN $(5,10$ or $15 \mu \mathrm{M}, 1 \mathrm{~h}$ ). AP1 was then added to the culture to co-treat for another $4 \mathrm{~h}$. Similar to the aforementioned fluorescent-labelling step, drug- or chemical-treated 
parasites were isolated from RBCs and lysed. Probe-labelled proteins were then subjected to rhodamine-azide conjugation, SDS-PAGE separation and fluorescence scanning.

For haem modulator treatment in early ring-stage parasites, highly synchronized early ring-stage parasites were pretreated with ALA $(1 \mathrm{mM})$ or SA $(0.5 \mathrm{mM})$ or with a combination of ALA $(1 \mathrm{mM})$ and SA $(0.5 \mathrm{mM})$ for $1 \mathrm{~h}$. AP1 was then added and incubated together with the haem modulators for another $4 \mathrm{~h}$. Probe-labelled proteins were then subjected to rhodamine-azide conjugation, SDS-PAGE separation and fluorescence scanning.

Parasite labelling for target identification. Five millilitres of parasite cultures (unsynchronized, 5\% parasitaemia, $1.25 \%$ haematocrit) were incubated with AP1 $(500 \mathrm{nM})$ for $4 \mathrm{~h}$ at $37^{\circ} \mathrm{C}$. Parasite culture containing $0.2 \%$ DMSO was used as a negative control. Similar to the above procedure for fluorescence scanning, parasites were isolated and purified for subsequent click chemistry to conjugate proteins with the biotin tag. For each reaction, biotin-azide $(10 \mu \mathrm{M})$, TCEP $(1 \mathrm{mM}$, $100 \times$ fresh stock in water), TBTA ligand $(100 \mu \mathrm{M}, 100 \times$ stock in DMSO $)$ and $\mathrm{CuSO}_{4}(1 \mathrm{mM}, 100 \times$ stock in water) were sequentially added to the cell lysates, and the lysates were incubated at room temperature for $4 \mathrm{~h}$ with shaking.

Then, clicked proteins were subjected to precipitation with acetone and air dried. Subsequently, the pellet was dissolved in $5 \mathrm{ml}$ of $0.1 \%$ SDS in PBS and incubated with $50 \mu \mathrm{l}$ of streptavidin beads (Sigma-Aldrich) under gentle mixing for $2 \mathrm{~h}$ at room temperature.

On-bead digestion by trypsin. Beads were washed thrice with $1 \%$ SDS, urea $(6 \mathrm{M})$ and PBS before being re-suspended in $100 \mu \mathrm{l}$ triethylammonium bicarbonate ( $25 \mathrm{mM}$ ), in which $2 \mu \mathrm{l}$ TCEP ( $100 \mathrm{mM}$ stock solution) was then added. The beads were heated at $65^{\circ} \mathrm{C}$ for $1 \mathrm{~h}$ before allowing the samples to react for $15 \mathrm{~min}$ at room temperature, after which $1 \mu \mathrm{l}$ MMTS (200 mM stock solution) was added. With reduction and alkylation performed, trypsin $\left(12.5 \mathrm{ng}^{-1}\right)$ was added to the samples, which were then incubated at $37^{\circ} \mathrm{C}$ overnight. The beads were separated from the digested peptides with a filter-spin column (GE Healthcare).

Liquid chromatography-mass spectrometry/mass spectrometry. The LC-MS/ MS method was described previously ${ }^{58}$. Separation of the peptides was performed using an Eksigent nanoLC Ultra and ChiPLC-nanoflex (Eksigent, Dublin, CA, USA) in Trap-Elute configuration. Desalting of the samples was carried out with a Sep-Pak tC $18 \mu$ l Elution Plate (Waters, Milford, MA, USA) before reconstituting in $50 \mu \mathrm{l}$ of diluent ( $98 \%$ water, $2 \% \mathrm{ACN}$ and $0.1 \% \mathrm{FA}$ ). With the use of a $200 \mu \mathrm{m} \times 0.5 \mathrm{~mm}$ trap column, $5 \mu \mathrm{l}$ of each sample was loaded and eluted on an analytical column $(75 \mu \mathrm{m} \times 150 \mathrm{~mm})$. Both trap and analytical columns were made of ChromXP C18-CL, $3 \mu \mathrm{m}$ (Eksigent, Germany). At a flow rate of $300 \mathrm{nl} \mathrm{min}{ }^{-1}$, the peptides were segregated by a gradient formed with mobile phase A $(2 \% \mathrm{ACN}$, $0.1 \% \mathrm{FA})$ and mobile phase B (98\% ACN, $0.1 \% \mathrm{FA}): 5-12 \%$ of mobile phase B ( $20 \mathrm{~min}), 12-30 \%$ of mobile phase B ( $90 \mathrm{~min}), 30-90 \%$ of mobile phase B ( $2 \mathrm{~min}$ ), $90 \%$ of mobile phase B $(5 \mathrm{~min}), 90-5 \%$ of mobile phase B $(3 \mathrm{~min})$ and $5-5 \%$ of mobile phase B (13 min).

MS analysis was carried out with a TripleTOF 5600 system (SCIEX, Foster City, CA, USA) in information-dependent mode. A high-resolution mode $(>30,000)$, which consists of 250 -ms accumulation time per spectrum and a mass range of $400-1,250 \mathrm{~m} / \mathrm{z}$, was set before MS spectra were obtained. For each duty cycle/MS spectrum, a maximum of 20 precursors with a charge state between 2 and 4 was selected for fragmentation, with a 100-ms minimum accumulation time for each precursor and dynamic exclusion for $15 \mathrm{~s}$.

The protein and peptide summary of artemisinin targets are shown in Supplementary Data $2-7$.

The mass spectrometry proteomics data were deposited in the ProteomeXchange Consortium ${ }^{59}$ via the PRIDE partner repository with the data set identifier PXD002611.

ProteinPilot analysis. The detailed methods of ProteinPilot analysis have been illustrated previously ${ }^{60}$. The proteins were identified with ProteinPilot 4.5 (SCIEX) that uses a Paragon algorithm to carry out database searches. PlasmoDB (v13, $P$. falciparum 3D7) was used as the database, while the search parameters used were as follows: cysteine alkylation with MMTS; trypsin digestion; TripleTOF 5600; and biological modifications. The identified proteins were grouped using the ProGroup algorithm in the software to remove any redundancy. To determine the falsediscovery rate (FDR) for protein identification, a decoy database search strategy was utilized, of which a corresponding randomized database was produced using the Proteomics System Performance Evaluation Pipeline feature in the ProteinPilot 4.5 software. An unused score $\geq 1.3$ was used as the cutoff threshold for protein identification, after which the FDR equals to 0 .

Mascot analysis. The mass spectrometric data were converted into Mascot generic format (MGF) using the ProteinPilot (SCIEX) software and then searched with Mascot 2.4.0 (Matrix Science). The database used was a combined database containing PlasmoDB (v13, P. falciparum 3D7) and human protein sequences (total of 95,492 sequences). The search parameters used were as follows: trypsin digestion; ESI-QUAD-TOF; acetyl ( $N$-rterm), oxidation (M) and methylthio (C) as variable modifications; peptide mass tolerance was 100 p.p.m. and fragment mass tolerance was $0.4 \mathrm{Da}$; the maximum number of missed cleavages was 1 . A decoy database search strategy was used to determine the FDR for peptide identification. We applied 1\% FDR to filter the identified peptide list.

\section{Cloning, expression and purification of recombinant $\boldsymbol{P}$. falciparum proteins.}

Purified genomic DNA from the P. falciparum 3D7 strain was obtained using an AxyPrep multisource genomic DNA miniprep kit (Corning, Tewksbury, MA, USA). The genes encoding $P$. falciparum $L D H^{61}, O A T^{62}, S A M S$ and $T C T P^{63}$, which do not have introns, were amplified by PCR directly using the genomic DNA as a template, while the genes encoding PyrK and SpdSyn were PCR amplified from the plasmids purchased from Addgene (PK_plasmid 25278 and SRM_plasmid 25110, Cambridge, MA, USA). The primers used for PCR amplification are listed in Supplementary Table 5. The PCR products were cloned into the pET28a vector (Novagen, Madison, WI, USA), and the encoded genes of interest in the constructs were confirmed by sequencing. To overexpress the recombinant proteins, the plasmids were then transformed into competent E. coli BL21 (DE3)-star cells (Invitrogen, Carlsbad, CA, USA). Ten millilitres of an overnight culture were diluted 1:100 in Luria-Bertani medium supplemented with $100 \mathrm{\mu g} \mathrm{ml}^{-1}$ ampicillin and grown at $37^{\circ} \mathrm{C}$ until the $\mathrm{OD}_{600}$ reached $\sim 0.6$. Expression of recombinant proteins was induced with $0.4 \mathrm{mM}$ isopropyl $\beta$-D-1-thiogalactopyranoside. After overnight cultivation at $20^{\circ} \mathrm{C}$, the cells were collected by centrifugation and then re-suspended in B-per bacterial protein extraction reagent (Pierce, Thermo Scientific, Rockford, IL, USA) to extract the soluble proteins. After centrifugation, the supernatant was applied onto a Ni-NTA column (Thermo Scientific). The column was washed with buffer A (50 mM Tris- $\mathrm{HCl}, 300 \mathrm{mM} \mathrm{NaCl}, \mathrm{pH} 7.4)$ followed by buffer B (buffer A with $10 \mathrm{mM}$ imidazole, $\mathrm{pH}$ 7.4). The recombinant proteins were eluted with buffer C (buffer A with $250 \mathrm{mM}$ imidazole, $\mathrm{pH}$ 7.4).

Validation of drug targets using western blots. The AP1 affinity pull-down sample was separated via one-dimensional SDS-PAGE together with the DMSO pull-down sample. After SDS-PAGE, the proteins were transferred onto polyvinylidene difluoride membranes (Bio-Rad). The blots were blocked with 5\% $(\mathrm{w} / \mathrm{v}) \mathrm{BSA}$ in PBS with $0.1 \%$ Tween 20 (PBS-T) for $4 \mathrm{~h}$ at room temperature. The membranes were incubated with rabbit anti-actin (1: 500; Sigma), rabbit anti-MSP-1 (1:5,000), mouse anti-PM-I (1:5,000) and rabbit anti-PM-II $(1: 5,000)$. Horseradish peroxidase (HRP)-conjugated anti-rabbit (Pierce Biotechnology) or HRP-conjugated anti-mouse IgG (1:5,000; GE Healthcare) was used as a secondary antibody, and samples were incubated for $2 \mathrm{~h}$ at room temperature. The membrane was washed three times in PBS-T between each antibody incubation step. Subsequent visualization was performed using ECL substrate (Pierce Biotechnology).

In vitro labelling of $\boldsymbol{P}$. falciparum recombinant proteins. Recombinant $\mathrm{OAT}$, TCTP, PyrK, SpdSyn, LDH and SAMS were reconstituted with PBS at $1 \mathrm{mg} \mathrm{ml}^{-1}$ Two microlitres of protein solution were used for labelling. To test the activation effects of AP1 $(20 \mu \mathrm{M})$ on the protein solution, a combination of different treatments involving haemin, $\mathrm{L}$-ascorbic acid $(\mathrm{Vc}), \mathrm{Na}_{2} \mathrm{~S}_{2} \mathrm{O}_{4}, \mathrm{FeSO}_{4}$ and DFO (200 $\mu \mathrm{M}$ each) was used. The final reaction volume was topped up to $40 \mu \mathrm{l}$ with PBS and incubated for $4 \mathrm{~h}$ at room temperature with shaking.

In this section, three main experiments were performed as follows. For concentration-dependent and time-dependent labelling, the proteins were treated with different doses of AP1 and incubation durations in the presence of haemin and Vc. For the heat-denatured samples, the protein solution was dissolved in 1\% SDS solution by diluting the sample with $1.2 \%$ SDS in PBS. After dissolving, the samples were heated at $96^{\circ} \mathrm{C}$ for $10 \mathrm{~min}$ before cooling down to room temperature. AP1 $(20 \mu \mathrm{M})$, haemin and $\mathrm{Vc}_{\mathrm{c}}$ were then added for labelling. A competition assay was carried out by pretreating the proteins with excess artesunate $(25 \times)$ for $1 \mathrm{~h}$ in the presence of haem (haemin reduced by $\mathrm{Vc}$ ) before incubating with AP1 together with artesunate for another $4 \mathrm{~h}$. To test whether the thiol and amine groups of the proteins react with the AP1 probe, proteins were pretreated with $30 \mathrm{mM}$ IAA or $10 \mathrm{mM} \mathrm{N}$-ethylmaleimide for $20 \mathrm{~min}$, and AP1 was added to react for another $4 \mathrm{~h}$

All the aforementioned treatments and labelling were stopped by adding four volumes of pre-chilled acetone. The unreacted probe and other chemicals were removed after acetone precipitation of the proteins. The resulting pellets were dissolved in $0.16 \%$ SDS in PBS and tagged with the fluorescent dye for SDS-PAGE and fluorescence scanning.

LDH inhibition assay. The assay followed the manufacturer's protocol with slight modifications. Purified LDH $(1 \mu \mathrm{g})$ was first incubated with haemin $(200 \mu \mathrm{M})$, Vc $(200 \mu \mathrm{M})$ and different concentrations of artesunate $(0-80 \mu \mathrm{M})$ in assay buffer $(50 \mu \mathrm{l})$ at $37^{\circ} \mathrm{C}$ for $4 \mathrm{~h}$. The resulting mixture was transferred to a 96-well plate, followed by the addition of the reaction mix containing assay buffer $(48 \mu \mathrm{l})$ and LDH substrate mix $(2 \mu \mathrm{l})$. LDH activity was subsequently measured by absorbance at $450 \mathrm{~nm}$ with a microplate reader (Infinite M200 PRO, Tecan, Maennedorf, Switzerland). The reactions with no LDH added and with LDH without inhibitor served as negative and positive controls, respectively. All measurements were carried out in triplicate. 
Pyruvate kinase inhibition assay. The assays followed the manufacturer's protocol with slight modifications. Purified pyruvate kinase $(1 \mu \mathrm{g})$ was first incubated with haemin $(200 \mu \mathrm{M}), \mathrm{Vc}(200 \mu \mathrm{M})$ and different concentrations of artesunate $(0-80 \mu \mathrm{M})$ in assay buffer $(50 \mu \mathrm{l})$ at $37^{\circ} \mathrm{C}$ for $4 \mathrm{~h}$. The resulting mixture was transferred to a 96-well plate, followed by addition of the reaction mix containing assay buffer $(44 \mu \mathrm{l})$, substrate mix $(2 \mu \mathrm{l})$, enzyme mix $(2 \mu \mathrm{l})$ and fluorescent peroxidase substrate $(2 \mu \mathrm{l})$. Pyruvate kinase activity was subsequently measured by absorbance at $570 \mathrm{~nm}$ with a microplate reader (Infinite M200 PRO, Tecan). The reactions with no pyruvate kinase added and pyruvate kinase without inhibitor served as negative and positive controls, respectively. All measurements were carried out in triplicate.

Pathway analysis. The GO analysis was conducted using Cytoscape 3.2.1 software ${ }^{64,65}$ with the ClueGO ${ }^{66}$ plugin. The database (GO Plasmodium falciparum) used for the GO analysis was obtained from the GO Consortium ${ }^{67}$. The GO analysis was conducted using a two-sided hypergeometric test with Bonferroni correction. The GO term levels were from five to ten. The minimum number of genes to form a cluster was set at two, while the minimum percentage of genes covered by our data set against the database was set at $10 \%$. The rest of the settings were left as defaults. The resultant Fig. 2 shows the major biological processes of the identified protein targets of artemisinin, while Supplementary Fig. 7 shows the cellular localization and Supplementary Fig. 8 shows the molecular functions.

Cancer cell (HCT 116) culture. The HCT116 cell line was obtained from ATCC (Manassas, VA, USA) and cultured in Dulbecco's modified eagle medium (Sigma-Aldrich) supplemented with $10 \%$ fetal bovine serum (Invitrogen) and $1 \times$ antibiotic/antimycotic (Invitrogen). Cells were cultured at $37^{\circ} \mathrm{C}$ supplemented with $5 \% \mathrm{CO}_{2}$

\section{Haem modulator treatment and in vitro killing assay of HCT116 cells.}

Approximately 20,000 HCT116 cells were plated in triplicate in a 96-well plate and incubated for $24 \mathrm{~h}$ before being subjected to pretreatment with AP1 and various haem modulators. A concentration of $35 \mu \mathrm{M}$ AP1 was used to treat the cells. For haem modulator pretreatment, $1 \mathrm{mM}$ ALA, $0.5 \mathrm{mM} \mathrm{SA}, 100 \mu \mathrm{M} \mathrm{FeSO}_{4}$ and $100 \mu \mathrm{M}$ DFO were added for $1 \mathrm{~h}$ before being incubated together with artesunate for another $24 \mathrm{~h}$. Cell viability was determined by the crystal violet assay as described previously ${ }^{68}$. Briefly, PBS was used to remove excess medium and dead cells that were still attached to the plate. The cells were then stained with $0.5 \%$ crystal violet in $20 \%$ methanol for 15 min. Excess crystal violet was washed off with PBS, and the plates were left to air dry. The cells were then solubilized with $1 \%$ SDS for $30 \mathrm{~min}$, and the absorbance was measured at $550 \mathrm{~nm}$. All the results (the cell survival rates) were normalized against the controls. The killing effect was calculated as $100 \%$ survival rate minus the respective cell survival rate of each treatment. All measurements were carried out in triplicate.

\section{Fluorescence scanning of HCT116 cells treated with the AP1 and haem}

modulators. HCT116 cells were cultured in a 12 -well plate until reaching $75 \%$ confluence. Thirty-five micromolar AP1 was used to treat the cells. Similar concentrations of haem modulators as mentioned in the killing assay (the section above) were used for the pretreatment ( $1 \mathrm{~h}$ ) and incubated together with AP1 for another $6 \mathrm{~h}$. The cells were washed three times with PBS to remove any free probe and other compounds present. Cells were then lysed by lysis buffer $(0.16 \%$ SDS in PBS) before the protein concentrations were determined with a DC protein assay (Bio-Rad). Approximately $20 \mu \mathrm{g}$ proteins for each treatment were clicked with rhodamine-azide for fluorescence scanning. The florescence intensity of the gel was quantified using ImageQuant. All measurements were carried out in triplicate.

\section{References}

1. Manske, M. et al. Analysis of Plasmodium falciparum diversity in natural infections by deep sequencing. Nature 487, 375-379 (2012).

2. Nosten, F. \& White, N. J. Artemisinin-based combination treatment of falciparum malaria. Am. J. Trop. Med. Hyg. 77, 181-192 (2007).

3. Meshnick, S. R. Artemisinin: mechanisms of action, resistance and toxicity. Int. J. Parasitol. 32, 1655-1660 (2002).

4. Meunier, B. \& Robert, A. Heme as trigger and target for trioxane-containing antimalarial drugs. Acc. Chem. Res. 43, 1444-1451 (2010).

5. Meshnick, S. R. et al. Iron-dependent free radical generation from the antimalarial agent artemisinin (qinghaosu). Antimicrob. Agents Chemother. 37, 1108-1114 (1993).

6. O’Neill, P. M., Barton, V. E. \& Ward, S. A. The molecular mechanism of action of artemisinin - the debate continues. Molecules 15, 1705-1721 (2010).

7. Robert, A., Cazelles, J. \& Meunier, B. Characterization of the alkylation product of heme by the antimalarial drug artemisinin. Angew. Chem. Int. Ed. 40, 1954-1957 (2001).
8. Asawamahasakda, W., Ittarat, I., Pu, Y. M., Ziffer, H. \& Meshnick, S. R. Reaction of antimalarial endoperoxides with specific parasite proteins. Antimicrob. Agents Chemother. 38, 1854-1858 (1994).

9. Eckstein-Ludwig, U. et al. Artemisinins target the SERCA of Plasmodium falciparum. Nature 424, 957-961 (2003).

10. Stocks, P. A. et al. Evidence for a common non-heme chelatable-irondependent activation mechanism for semisynthetic and synthetic endoperoxide antimalarial drugs. Angew. Chem. Int. Ed. 46, 6278-6283 (2007).

11. Klonis, N. et al. Artemisinin activity against Plasmodium falciparum requires hemoglobin uptake and digestion. Proc. Natl Acad. Sci. USA 108, 11405-11410 (2011).

12. Bhisutthibhan, J. et al. The Plasmodium falciparum translationally controlled tumor protein homolog and its reaction with the antimalarial drug artemisinin. J. Biol. Chem. 273, 16192-16198 (1998).

13. Paulick, M. \& Bogyo, M. Application of activity-based probes to the study of enzymes involved in cancer progression. Curr. Opin. Genet. Dev. 18, 97-106 (2008).

14. Nomura, D. K., Dix, M. M. \& Cravatt, B. F. Activity-based protein profiling for biochemical pathway discovery in cancer. Nat. Rev. Cancer 10, 630-638 (2010).

15. Willems, L. I. et al. Bioorthogonal chemistry: applications in activity-based protein profiling. Acc. Chem. Res. 44, 718-729 (2011).

16. Speers, A. E., Adam, G. C. \& Cravatt, B. F. Activity-based protein profiling in vivo using a copper(I)-catalyzed azide-alkyne $[3+2]$ cycloaddition. J. Am. Chem. Soc. 125, 4686-4687 (2003).

17. Ovaa, H. et al. Chemistry in living cells: detection of active proteasomes by a two-step labeling strategy. Angew. Chem. Int. Ed. 115, 3754-3757 (2003).

18. Evans, M. J. \& Cravatt, B. F. Mechanism-based profiling of enzyme families. Chem. Rev. 106, 3279-3301 (2006).

19. Fonović, M. \& Bogyo, M. Activity based probes as a tool for functional proteomic analysis of proteases. Expert Rev. Proteomics 5, 721-730 (2008).

20. Böttcher, T., Pitscheider, M. \& Sieber, S. A. Natural products and their biological targets: proteomic and metabolomic labeling strategies. Angew. Chem. Int. Ed. 49, 2680-2698 (2010).

21. Yang, P.-Y. et al. Activity-based proteome profiling of potential cellular targets of Orlistat-an FDA-approved drug with anti-tumor activities. J. Am. Chem. Soc. 132, 656-666 (2010)

22. Liu, C.-X. et al. Adenanthin targets peroxiredoxin I and II to induce differentiation of leukemic cells. Nat. Chem. Biol. 8, 486-493 (2012).

23. Ariey, F. et al. A molecular marker of artemisinin-resistant Plasmodium falciparum malaria. Nature 505, 50-55 (2013).

24. Wunderlich, J., Rohrbach, P. \& Dalton, J. P. The malaria digestive vacuole. Front. Biosci. 4, 1424-1448 (2012).

25. Mauritz, J. M. A. et al. The homeostasis of Plasmodium falciparum-infected red blood cells. PLoS Comput. Biol. 5, e1000339 (2009).

26. Ying-Zi, Y., Asawamahasakda, W. \& Meshnick, S. R. Alkylation of human albumin by the antimalarial artemisinin. Biochem. Pharmacol. 46, 336-339 (1993).

27. Berger, B. J. Antimalarial activities of aminooxy compounds. Antimicrob. Agents Chemother. 44, 2540-2542 (2000).

28. Sullivan, S. G., Baysal, E. \& Stern, A. Inhibition of hemin-induced hemolysis by desferrioxamine: binding of hemin to red cell membranes and the effects of alteration of membrane sulfhydryl groups. Biochim. Biophys. Acta 1104, 38-44 (1992).

29. O’Brien, C., Henrich, P. P., Passi, N. \& Fidock, D. A. Recent clinical and molecular insights into emerging artemisinin resistance in Plasmodium falciparum. Curr. Opin. Infect. Dis. 24, 570-577 (2011).

30. Reeder, B. J. \& Wilson, M. T. Desferrioxamine inhibits production of cytotoxic heme to protein cross-linked myoglobin: a mechanism to protect against oxidative stress without iron chelation. Chem. Res. Toxicol. 18, 1004-1011 (2005).

31. Reeder, B. J., Hider, R. C. \& Wilson, M. T. Iron chelators can protect against oxidative stress through ferryl heme reduction. Free Radic. Biol. Med. 44, 264-273 (2008).

32. Antoine, T. et al. Rapid kill of malaria parasites by artemisinin and semisynthetic endoperoxides involves ROS-dependent depolarization of the membrane potential. J. Antimicrob. Chemother. 69, 1005-1016 (2014).

33. Yang, N.-D. et al. Artesunate induces cell death in human cancer cells via enhancing lysosomal function and lysosomal degradation of ferritin. J. Biol. Chem. 289, 33425-33441 (2014).

34. Morel, I. et al. Antioxidant and free radical scavenging activities of the iron chelators pyoverdin and hydroxypyrid-4-ones in iron-loaded hepatocyte cultures: comparison of their mechanism of protection with that of desferrioxamine. Free Radic. Biol. Med. 13, 499-508 (1992).

35. Mercer, A. E., Copple, I. M., Maggs, J. L., O’Neill, P. M. \& Park, B. K. The role of heme and the mitochondrion in the chemical and molecular mechanisms of mammalian cell death induced by the artemisinin antimalarials. J. Biol. Chem. 286, 987-996 (2011).

36. Zhang, S. \& Gerhard, G. S. Heme mediates cytotoxicity from artemisinin and serves as a general anti-proliferation target. PLoS ONE 4, e7472 (2009). 
37. Ter Kuile, F., White, N., Holloway, P., Pasvol, G. \& Krishna, S. Plasmodium falciparum: in vitro studies of the pharmacodynamic properties of drugs used for the treatment of severe malaria. Exp. Parasitol. 76, 85-95 (1993).

38. Klonis, N. et al. Altered temporal response of malaria parasites determines differential sensitivity to artemisinin. Proc. Natl Acad. Sci. USA 110, 5157-5162 (2013).

39. Bakar, N. A., Klonis, N., Hanssen, E., Chan, C. \& Tilley, L. Digestive-vacuole genesis and endocytic processes in the early intraerythrocytic stages of Plasmodium falciparum. J. Cell Sci. 123, 441-450 (2010).

40. Nagaraj, V. A. et al. Malaria parasite-synthesized heme is essential in the mosquito and liver stages and complements host heme in the blood stages of infection. PLoS Pathog. 9, e1003522 (2013).

41. Komatsuya, K. et al. Synergy of ferrous ion on 5 -aminolevulinic acid-mediated growth inhibition of Plasmodium falciparum. J. Biochem. 154, 501-504 (2013).

42. Smith, T. G. \& Kain, K. C. Inactivation of Plasmodium falciparum by photodynamic excitation of heme-cycle intermediates derived from deltaaminolevulinic acid. J. Infect. Dis. 190, 184-191 (2004).

43. Sigala, P. a., Crowley, J. R., Henderson, J. P. \& Goldberg, D. E. Deconvoluting heme biosynthesis to target blood-stage malaria parasites. Elife 4, 1-20 (2015).

44. Sigala, P. A. \& Goldberg, D. E. The peculiarities and paradoxes of Plasmodium heme metabolism. Annu. Rev. Microbiol. 68, 259-278 (2014).

45. Ke, H. et al. The heme biosynthesis pathway is essential for plasmodium falciparum development in mosquito stage but not in blood stages. J. Biol. Chem. 289, 34827-34837 (2014).

46. Dondorp, A. M. et al. Artemisinin resistance in Plasmodium falciparum malaria. N. Engl. J. Med. 361, 455-467 (2009).

47. Ashley, E. A. et al. Spread of artemisinin resistance in Plasmodium falciparum malaria. N. Engl. J. Med. 371, 411-423 (2014).

48. Mok, S. et al. Population transcriptomics of human malaria parasites reveals the mechanism of artemisinin resistance. Science 347, 431-435 (2015).

49. Straimer, J. et al. K13-propeller mutations confer artemisinin resistance in Plasmodium falciparum clinical isolates. Science 347, 428-431 (2015).

50. Afonso, A. et al. Malaria parasites can develop stable resistance to artemisinin but lack mutations in candidate genes atp6 (encoding the sarcoplasmic and endoplasmic reticulum $\mathrm{Ca} 2+$ ATPase), tctp, mdr1, and cg10. Antimicrob. Agents Chemother. 50, 480-489 (2006).

51. Dogovski, C. et al. Targeting the cell stress response of Plasmodium falciparum to overcome artemisinin resistance. PLoS Biol. 13, el002132 (2015).

52. Krammer, B. \& Plaetzer, K. ALA and its clinical impact, from bench to bedside. Photochem. Photobiol. Sci. 7, 283-289 (2008).

53. Mbengue, A. et al. A molecular mechanism of artemisinin resistance in Plasmodium falciparum malaria. Nature 520, 683-687 (2015).

54. Liu, Y. et al. Synthesis and antimalarial activity of novel dihydro-artemisinin derivatives. Molecules 16, 4527-4538 (2011).

55. O'Neill, P. M. et al. Optimisation of the allylsilane approach to C-10 deoxo carba analogues of dihydroartemisinin: synthesis and in vitro antimalarial activity of new, metabolically stable C-10 analogues. J. Chem. Soc. Perkin Trans. $1,2682-2689$ (2001).

56. Liu, Y., Wong, V. K. W., Ko, B. C. B., Wong, M. K. \& Che, C. M. Synthesis and cytotoxicity studies of artemisinin derivatives containing lipophilic alkyl carbon chains. Org. Lett. 7, 1561-1564 (2005).

57. Chadwick, J., Mercer, A. E., Park, B. K., Cosstick, R. \& O’Neill, P. M. Synthesis and biological evaluation of extraordinarily potent C-10 carba artemisinin dimers against P. falciparum malaria parasites and HL-60 cancer cells. Bioorg. Med. Chem. 17, 1325-1338 (2009).

58. Tang, L. A. L. et al. High-performance graphene-titania platform for detection of phosphopeptides in cancer cells. Anal. Chem. 84, 6693-6700 (2012).

59. Vizcaíno, J. A. et al. ProteomeXchange provides globally coordinated proteomics data submission and dissemination. Nat. Biotechnol. 32, 223-226 (2014).

60. Ghosh, D. et al. Identification of key players for colorectal cancer metastasis by iTRAQ quantitative proteomics profiling of isogenic SW480 and SW620 cell lines. J. Proteome Res. 10, 4373-4387 (2011).
61. Kehr, S. et al. Protein S-glutathionylation in malaria parasites. Antioxid. Redox Signal. 15, 2855-2865 (2011).

62. Jortzik, E. et al. Redox regulation of Plasmodium falciparum ornithine $\delta$ aminotransferase. J. Mol. Biol. 402, 445-459 (2010).

63. Eichhorn, T. et al. Molecular interaction of artemisinin with translationally controlled tumor protein (TCTP) of Plasmodium falciparum. Biochem. Pharmacol. 85, 38-45 (2013).

64. Cline, M. S. et al. Integration of biological networks and gene expression data using Cytoscape. Nat. Protoc. 2, 2366-2382 (2007).

65. Saito, R. et al. A travel guide to Cytoscape plugins. Nat. Methods 9, 1069-1076 (2012).

66. Bindea, G. et al. ClueGO: a Cytoscape plug-in to decipher functionally grouped gene ontology and pathway annotation networks. Bioinformatics 25, 1091-1093 (2009).

67. Ashburner, M. et al. Gene Ontology: tool for the unification of biology. Nat. Genet. 25, 25-29 (2000).

68. Wang, J. et al. A quantitative chemical proteomics approach to profile the specific cellular targets of andrographolide, a promising anticancer agent that suppresses tumor metastasis. Mol. Cell. Proteomics 13, 876-886 (2014).

\section{Acknowledgements}

We thank Dr Chengchao Xu, Dr Cynthia He and Dr Chu-Young Kim from the National University of Singapore for their critical review of this manuscript. This work was funded in part by the National Research Foundation-supported Interdisciplinary Research group in Infectious Diseases of SMART (Singapore-MIT Alliance for Research and Technology).

\section{Author contributions}

J.W. conceived the idea for this project, designed and conducted experiments, and wrote the manuscript with contributions from other authors; C.-J.Z. and J.W. designed the probes and conducted the in vitro binding experiments; W.N.C., C.C.Y.L., M.L., Y.Q.L. and C.T.L. provided the parasites and tested the drugs' killing effects; Z.L., L.-X.Y., N.L. and C.X.L expressed the target proteins; Y.K.H., J.Z., Z.C.H, B.L and H.-M.S. assisted in the data analysis; Y.M.L performed the Gene Ontology analysis; T.K.L. assisted in target identification; K.S.W.T. and Q.L supervised and supported the project; all authors discussed the results and commented on the manuscript.

\section{Additional information}

Accession codes: The mass spectrometry proteomics data have been deposited in the ProteomeXchange Consortium via the PRIDE partner repository with the data set identifier PXD002611

Supplementary Information accompanies this paper at http://www.nature.com/ naturecommunications

Competing financial interests: The authors declare no competing financial interests.

Reprints and permission information is available online at http://npg.nature.com/ reprintsandpermissions/

How to cite this article: Wang, J. et al. Haem-activated promiscuous targeting of artemisinin in Plasmodium falciparum. Nat. Commun. 6:10111 doi: 10.1038/ ncomms10111 (2015)

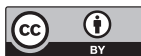

This work is licensed under a Creative Commons Attribution 4.0 International License. The images or other third party material in this article are included in the article's Creative Commons license, unless indicated otherwise in the credit line; if the material is not included under the Creative Commons license, users will need to obtain permission from the license holder to reproduce the material To view a copy of this license, visit http://creativecommons.org/licenses/by/4.0/ 